\title{
HYBRID FEATURE SELECTION FOR MYOELECTRIC SIGNAL CLASSIFICATION USING MICA
}

\author{
Ganesh R. Naik - Dinesh K. Kumar *
}

\begin{abstract}
This paper presents a novel method to enhance the performance of Independent Component Analysis (ICA) of myoelectric signal by decomposing the signal into components originating from different muscles. First, we use Multi run ICA (MICA) algorithm to separate the muscle activities. Pattern classification of the separated signal is performed in the second step with a back propagation neural network. The focus of this work is to establish a simple, yet robust system that can be used to identify subtle complex hand actions and gestures for control of prosthesis and other computer assisted devices. Testing was conducted using several single shot experiments conducted with five subjects. The results indicate that the system is able to classify four different wrist actions with near $100 \%$ accuracy.
\end{abstract}

K e y w o r d s: blind source separation (BSS), independent component analysis (ICA), surface electromyogram (sEMG), myoelectric signal (MES), source separation, human computer interface (HCI)

\section{INTRODUCTION}

Hand actions and maintained gestures are a result of complex combination of contraction of multiple muscles in the forearm. Myoelectric signal (MES) is the electrical recording of the muscle activity from the surface. It is closely related to the strength of muscle contraction and an obvious choice for control of the prosthesis and similar applications. MES is the electrical manifestation of muscular contraction. MES classification is one of the most difficult pattern recognition problems because there usually exist large variations in surface Electromyogram (sEMG) features. The sEMG signal has been used as a tool to provide advanced man-machine interfaces [1], rehabilitation of the handicapped people, functional electrical stimulation devices (FES) [2] and control commands for limb prostheses [3]. The classification problem may be divided into three steps:

(i) signal presentation,

(ii) feature extraction and

(iii) pattern recognition.

It is shown in this paper that classification performance of wrist movements depends upon method of classification and the choice of ICA algorithm. Many researches proposed several method of classification that showed good performance $[4,5,6]$.

Many attempts have been made to use sEMG signal as the command to control the prosthesis $[7,8]$, but none of them takes explicit advantage of its subtlety, the fact that commands can be issued without the generation of strong contraction and observable movements. Since all these muscles present in the forearm are close to each other, myo-electric activity observed from any muscle site comprises the activity from the neighbouring muscle as well, referred to as cross-talk. The cross-talk problem is more significant when the muscle activation is relatively weak (subtle) because the comparable signal strength is very low. Extraction of the useful information from such kind of sEMG becomes difficult mainly due to the low signal to noise ratio. At low level of contraction, EMG activity is hardly discernible from the background activity. To identify the small movements and gesture of the hand, there is need for identifying components of sEMG originating from the different muscles. There is little or no prior information of the muscle activity, and the signals have temporal and spectral overlap, making the problem suitable for blind source separation (BSS).

There are number of BSS techniques such as Independent component analysis (ICA) has found numerous applications in audio and biosignal processing disciplines. Research that isolates motor unit action potential (MUAP) originating from different muscles and motor units has been reported in 2004 [9]. Recently surface EMG with ICA has been proposed for the hand gesture identification [10]. Muscle activity originating from different muscles can be considered to be independent, and this gives an argument to use BSS methods for separation of muscle activity originating from the different muscles. The spatial location of the active muscle activity is the determining factor of the hand action and gesture. One technique that has been reported is the use of prior knowledge of the anatomy. The advantage of this approach is that the model based BSS removes ambiguity of the order and magnitude.

In the previous research ICA had been used for hand gesture identification using constant mixing matrix where the overall accuracy was reported $100 \%$ [10], but the number of hand gesture identification was restricted to three. This paper reports improving the identification of various hand gesture using multi run ICA (MICA) of sEMG. ICA algorithm was performed several times; at each instance mixing matrix was computed. Best mixing

* School of Electrical and Computing Engineering, RMIT University Melbourne, Australia, ganesh.naik@rmit.edu.au, dinesh@rmit.edu.au 
matrix was chosen based on the highest Signal to interference Ratio (SIR) of global matrix. The processing in this new input system consists of three major stages: At first, hand gestures are sensed from non-invasive surface electromyograms, and in the second step the activities of the involved individual muscles are decomposed by semiblind ICA. In the last step, the particular hand action is identified with an artificial neural network (ANN)

\section{HAND GESTURE IDENTIFICATION FOR HCI}

Human hand gestures are means of non-verbal interaction among people. They range from simple actions of pointing at objects to the more complex ones that express our feelings and communicate with others. The main applications of gesture recognition are communicative and manipulative. Some of the examples of applications include:

- Control of consumer electronics

- Interaction with visualization systems

- Control of mechanical systems

- Computer games

- Prosthetic control

- Rehabilitation for elderly and disabled

Numerous approaches have been applied to the problem of visual interpretation of gestures for Human Computer Interface (HCI). These include the use of visual [11], mechanical sensor [12] and sEMG based. Many of those approaches have been chosen and implemented to focus on a particular aspect of gestures: Hand tracking, pose classification, or hand posture interpretations $[13,14]$.

The use of mechanical sensors is the simplest to implement and devices such as sensor glove has been widely used [15]. However such a system is not suitable to provide the user a natural interaction. Vision based techniques have the limitation that these require restricted backgrounds and camera positions and are only suitable for a small set of gestures performed with only one hand [15]. Surface EMG based system is very attractive because it is non-invasive, economical, and provides the natural seamless connectivity to the user. The shortcomings in sEMG based systems have been the lack of reliability for small and subtle actions. This paper reports research conducted to identify maintained hand gesture based on the muscle activity by the decomposition of sEMG. It is a combining model based approach with blind source separation technique.

\section{SURFACE ELECTROMYOGRAPHY AND ICA}

Surface EMG is the electrical recording of the spatial and temporal integration of the MUAP originating from different motor units. The main purpose for the interest electromyography (EMG) signal is clinical application. It is usually used clinically for the diagnosis of neurological and neuromuscular problems. EMG is also used in many types of research laboratories, including those involved in biomechanics, motor control, neuromuscular physiology, movement disorders, postural control, and physical therapy. EMG is controlled by nervous system and depends on anatomical and psychological properties of muscles. It is an electrical signal acquired from different organs. EMG is usually a function of time, described in terms of amplitude, frequency and phase [16].

Electromyography (EMG) signals classification and processing can be used for varieties of clinical/biomedical applications, spectral pattern classification of intensitybased analysis, and modern human computer interaction. EMG signals acquired from muscles require advanced methods for detection, decomposition, processing, and classification. One property of sEMG is that the electrical activity of one muscle can generally be considered to be independent of other bioelectric signals such as electrocardiogram (ECG), electro-oculargram (EOG), and signals from neighbouring muscles. This opens an opportunity for using BSS methods for this application

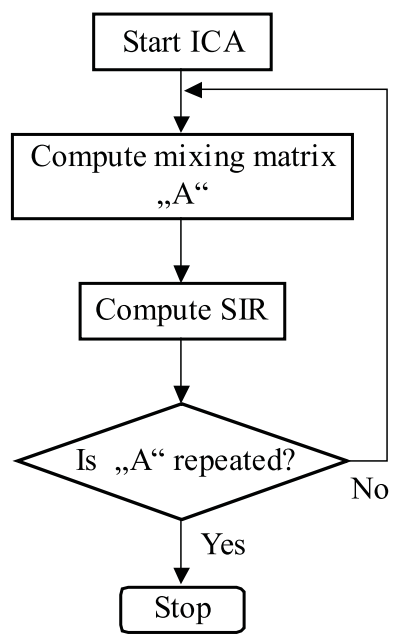

Fig. 1. MICA block diagram

\subsection{ICA model}

ICA is one of the widely used BSS technique, consists in recovering unobserved signals or 'sources' from several observed mixtures. Typically the observations are obtained at the output of a set of sensors, each sensor receiving the different combination of source signals. The simplest BSS technique aims at transforming an input vector into a signal space in which the signals are statistically independent [17].

The simplest ICA model assumes that the mixing process as linear, so it can be expressed as

$$
x(t)=A s(t)
$$

where $x(t)=\left[x_{1}(t), \ldots, x_{n}(t)\right]^{\top}$ are the recordings, $s(t)=\left[s_{1}(t), \ldots, s_{n}(t)\right]$ the original signals, and $A$ is the $n \times n$ mixing matrix. This mixing matrix and each of 
the original signals are unknown. To separate the recordings to the original signals (estimated original signals) the task is to estimate an un-mixing matrix $W$ so that

$$
s=W x(t)=W A s(t) .
$$

BSS is a difficult task because we do not have any information about the sources and the mixing process. BSS is a method to tackle this problem and is based on the assumption that the sources are independent from each other $[17,18]$. BSS iteratively determines the un-mixing matrix $W$ and thus estimates the corresponding independent signals $u$ from the observations $x$. There are number of possible cost-functions that may be considered for making the separated signals as independent as possible. The fundamental of these is based on the statistical independence of the sources $s$.

\subsubsection{Multi run ICA}

One of the most effective ways of modeling vector data for unsupervised pattern classification or coding is to assume that the observations are the result of picking randomly out of a fixed set of different distributions. ICA is an iterative BSS technique. At each instance original signals are estimated from the mixed data. The estimation quality depends mainly on the mixing matrix $A$.

Multi run ICA (MICA) is the process where the ICA algorithm will be computed many times; at each instance different mixing matrices will be obtained. $A_{1}, A_{2}, \ldots, A_{n}$. Since it is an iterative technique repeat analysis yields similarity matrices at some stage. Hence mixing matrices $A_{1}, A_{2}$ will repeat after certain iterations. The MICA process is shown in the Fig. 1.

MICA results in several matrices. To estimate the sources from the mixed data ICA requires just one mixing matrix, hence the best matrix has to be selected among the set of these mixing matrices, in order to yield better results. There are several methods to compute the quality of the mixing matrices. But signal to interference ratio (SIR) is a popular tool to perform this task [19].

This paper uses this unique technique to compute the mixing matrix from the sEMG signals. The paper also reports the improvement of hand gesture recognition performance.

\section{METHODOLOGY}

Experiments were conducted to evaluate the performance of the proposed hand gesture recognition system from hand muscle surface EMG. We have proposed a technique to classify small level of muscle activity to identify hand gesture using a combination of multi run Blind source separation (BSS), known muscle anatomy and neural network configured for the individual.

\subsection{Data Acquisition}

Four types of wrist movements to be classified are selected (Refer Fig. 2):

- Wrist flexion (G1)

- Wrist flexion towards little finger (G2)

- Wrist flexion towards thumb (G3) and

- Wrist and finger flexion together (G4).

The placement of EMG surface electrodes on muscle groups is important to have more information about each wrist movement. Four EMG surface electrodes are placed on four muscle groups, Brachioradialis (channel_1), Flexor Carpi radialis (channel_2), Flexor Carpi Ulnaris (channel_3) and extensor digitorum (channel_4), the locations of electrodes on one of the subject's arm is given in Fig. 3, from the input feature space, the classifier must be able to classify the four output classes exploiting the EMG signals measurements.

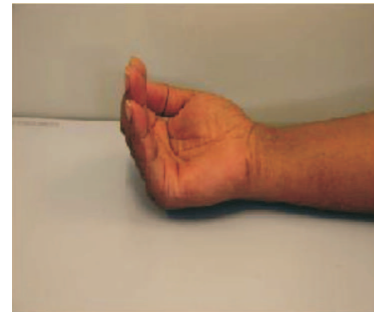

(a) - wrist flexion

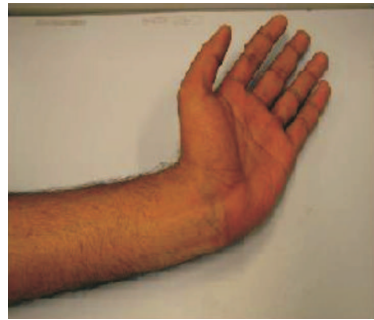

(c) - flexion towards little thumb

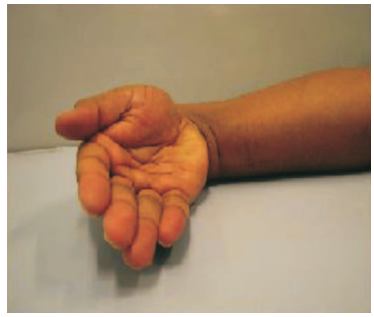

(b) - flexion towards little finger

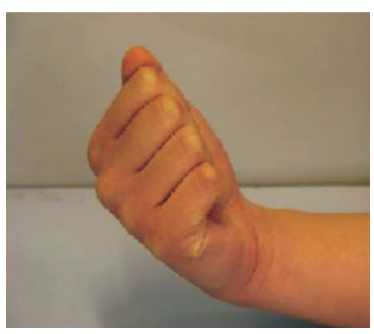

(d) - wrist and fingers together
Fig. 2. Four wrist actions performed during the experiment

Each channel has a pair of electrodes mounted together with a fixed inter-electrode distance of $10 \mathrm{~mm}$ and a gain of 1000. For each channel the signal was acquired using pair of electrodes with a fixed inter-electrode distance of $10 \mathrm{~mm}$ and a gain of 1000 . The signal was sampled at a rate of 1024 samples/sec using Delsys eight channel sEMG acquisition system (Boston, MA, USA).

The subjects were asked to produce 4 different wrist actions. The experiments were repeated on two different days. The forearm was resting on the table with elbow at an angle of approximately 90 degree and in a comfortable position. Four wrist and finger flexions were performed and each was repeated for a total of 24 times for each action over the two sessions. These wrist actions were selected for the experiment because these required four multiple muscles to be contracting at the same time and thus could test the ability of the system and this ensured that the estimated unmixing matrix was square for ICA 


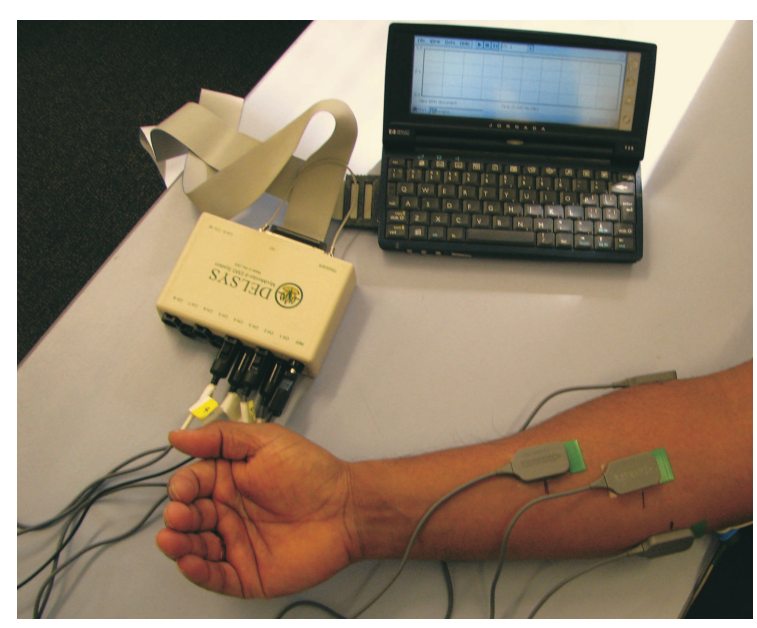

Fig. 3. Wrist action experimental set up with four electrodes

Table 1. SIR values for multi run ICA

\begin{tabular}{cc}
\hline Multi run ICA trials & SIR values \\
\hline 1 & 18.7348 \\
2 & 12.8453 \\
3 & 10.3121 \\
4 & 22.7227 \\
5 & 25.4145 \\
6 & 28.3418 \\
7 & 43.2347 \\
8 & 24.4565 \\
9 & 31.9706 \\
10 & 29.8656 \\
\hline
\end{tabular}

analysis. Markers were used to obtain the isometric contraction signals during recording. A suitable resting time was given between each experiment. There was no external load. These hand actions and gestures represented low level of muscle activity.

\subsection{Data analysis}

The aim of these experiments was to test the use of BSS algorithm [9] along with known properties of the muscles for separating muscle activity from sEMG recordings for the purpose of identifying subtle hand gestures. BSS methods are suitable when the numbers of recordings are same as or greater than the number of sources. Each experiment lasted approximately 2.5 seconds and was repeated 12 times. The sampling rate was 1024 samples per second. There were four channel (recordings) electrodes and four active muscles associated with the hand gesture, forming a square $4 \times 4$ mixing matrix. Mixing matrix was computed for the first set of data only and kept constant throughout the experiment. The mixing matrix $A$ was computed based on the multi run ICA. ICA algorithm was computed many times, at each instance SIR of mixing matrices were computed. Among them the best mix- ing matrix was chosen. The SIR computation process is explained next.

\subsubsection{SIR computation}

SIR for the mixing matrix $A$, performance index could be used for full-rank or non-full rank analysis. In view of the problem of one component estimation, we have

$$
y_{i}=w_{i}^{\top} X=\left(w_{i}^{\top} A\right) S=g_{i} S=g_{i j} s_{j}
$$

where $y_{i}$ and $s_{j}$ are the estimated component and the $j$-th source, respectively; $w_{i}^{\top}$ is a row vector of un-mixing matrix $W, g_{i}$ is a normalized row vector $\left[\begin{array}{lllll}0 & 0 & g_{i j} & 0 & 0\end{array}\right]$. Because $y_{i}$ is the estimation of $s_{j}$, the ideal normalized vector $g_{i}$ is the unit vector $u_{j}=$ $\left[\begin{array}{llll}0 & 0 & \ldots & 1 \ldots 0\end{array}\right]$. Therefore, one analysis is successful if and only if its vector $g_{i}$ is similar to one unit vector $u_{j}$.

Actually, vector $g_{I}$ is one row of matrix $G$. So, the quality of each estimated component just depends on one row of matrix $G$. The more different each row of $G$ is to each corresponding unit vector of $R^{N x N}$, the less quality of output we have. The SIR of each mixing matrix was computed using the following expression which evaluates the success of one component separation [19].

$$
S I R_{-} g=-10 \log 10\left(\left\|g_{i}-u_{j}\right\|_{2}^{2}\right) .
$$

The SIR values for the MICA algorithm for hand gesture experiments are shown in Table 1.

\subsection{RMS feature extraction}

The best mixing matrix was selected based on the highest SIR of mixing matrix. The selected mixing matrix was kept constant throughout the experiment. The independent sources of motor unit action potentials that mix to make the EMG recordings were estimated using the following equation.

$$
s=W x
$$

where, $W$ is the inverse of the mixing matrix A. This process was repeated for each of the four hand gesture experiments. Four sources were estimated for each experiment. After separating the four sources sa, $s b, s c$ and $s d$, each of these was segmented to 2500 samples length. Root Mean Squares (RMS) was computed for each of the separated sources using the following relation.

$$
S_{r m s}=\sqrt{\frac{1}{N} \sum_{i=1}^{n} s_{i}^{2}}
$$

where $s$ are the estimated sources and $N$ is the number of samples $(N=2500)$. This results in one number representing the muscle activity for each channel for each hand action. RMS value of muscle activity of each source represents the muscle activity of that muscle and is indicative of the strength of contraction. The above process was repeated for all four different hand actions 12 times and for each of the participants. These 12 sets of examples were used to train a back-propagation neural network. 


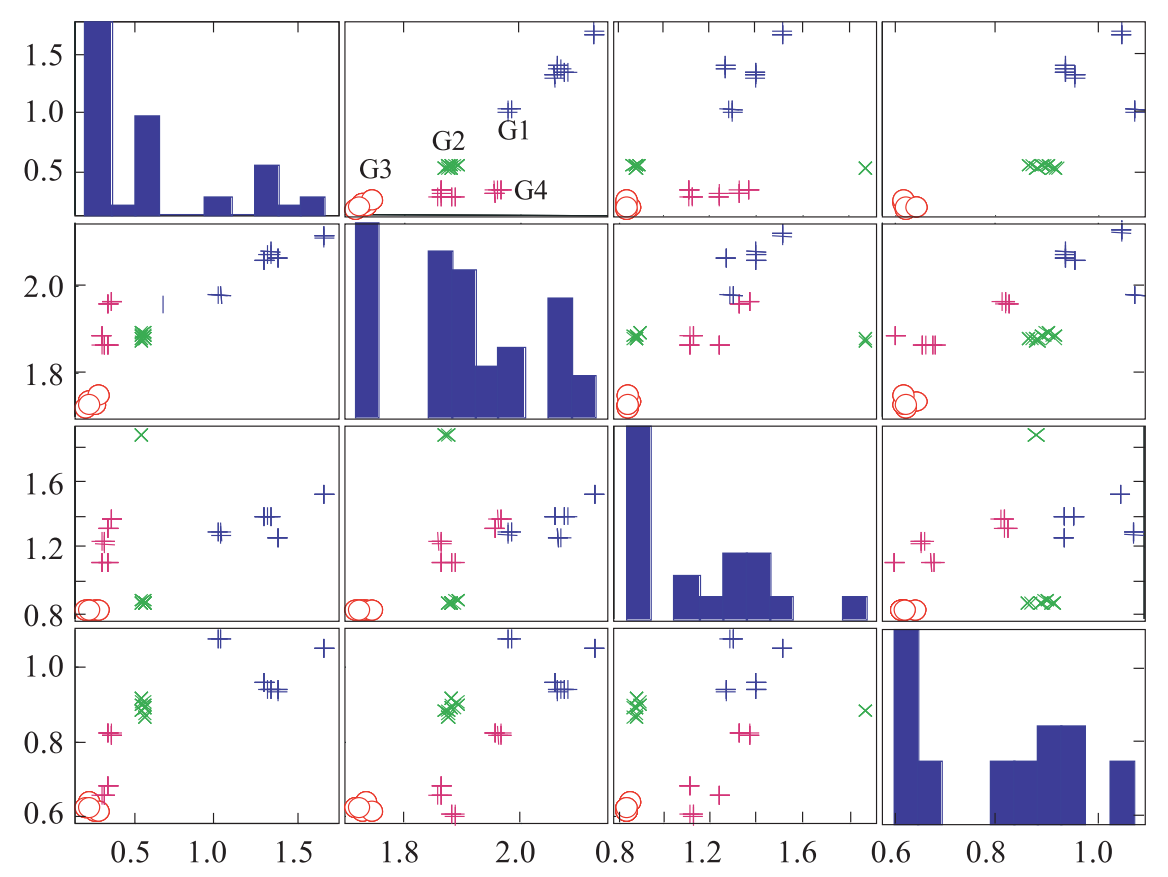

Fig. 4. Grouped plot of four channels MICA separated RMS features

Table 2. MANOVA table for 4 Channel data for four different wrist movements

\begin{tabular}{ccccc}
\hline & Criterion & Test Statistic & $\mathrm{F}$ & $\mathrm{P}$ \\
\hline Subject 1 & Wilk's & 0.00454 & 148.464 & 0.000 \\
& Lawley Hotelling & 37.99736 & 304.200 & 0.000 \\
& Pillai's & 1.688913 & 50.177 & 0.000 \\
\hline Subject 2 & Wilks' & 0.00571 & 138.798 & 0.000 \\
& Lawley-Hotelling & 28.95460 & 266.278 & 0.000 \\
& Pillai's & 1.93257 & 47.978 & 0.000 \\
\hline Subject 3 & Wilk's & 0.01665 & 89.866 & 0.000 \\
& Lawley Hotelling & 19.99307 & 167.937 & 0.000 \\
& Pillai's & 1.71030 & 37.131 & 0.000 \\
\hline Subject 4 4 Wilks' & 0.00102 & 289.273 & 0.000 \\
& Lawley-Hotelling & 53.03187 & 464.273 & 0.000 \\
& Pillai's & 2.46329 & 98.650 & 0.000 \\
\hline Subject 5 & Wilks' & 0.00324 & 169.427 & 0.000 \\
& Lawley-Hotelling & 43.7216 & 324.342 & 0.000 \\
& Pillai's & 2.02437 & 56.754 & 0.000 \\
\hline
\end{tabular}

\subsection{Preliminary Data Analysis using MANOVA}

Prior to the data classification the preliminary analysis was conducted to identify the separation of the data using grouped plot of for various combination and Multivariate Analysis of Variance (MANOVA). Figure 4 shows the grouped plot for MICA RMS features. From the figure it is evident that there is clear class separation among the four different gestures. Further, results verified statistically using MANOVA.

MANOVA is an extension of One-Way Analysis of Variance (ANOVA) and is suitable for analyzing more than one dependent variable. MANOVA measures the differences for two or more metric dependent variables based on a set of categorical variables acting as independent variables [20]. This was used to determine the significance of separation of the different classes of the data. The MANOVA analysis showed the statistical significance of the relationship of MICA with muscle contraction for different wrist actions. The overall statistical results for the different participants have been tabulated in Tab. 2. From Tab. 2 it can be observed that the $p$-value $(p<0.0001)$, are identifiable using MICA RMS features.

\subsection{Pattern recognition}

Hudgins' identification of nonrandom structure in the myoelectric signal at the onset of a muscle contraction suggests that pattern-recognition techniques may be usefully applied to the myoelectric control problem. If the myoelectric signal patterns caused by a variety of voluntary muscle motions can be reliably identified, they can be used to control prosthetic devices. Inherent to the process of pattern recognition is some form of classification decision in which input data are assigned to a limited number of distinct classes. Data samples within the same class are assumed to have one or more specific features in common which would cause them to be placed in the same class. This basic classification-process model is used in several approaches which have been developed to analyze the structure of the myoelectric signal. Of relevance to the current work are approaches which take advantage of the unique computational abilities of artificial neural networks (ANN) to perform the classification task. ANNbased classifiers are able to classify input data into distinct classes by "learning" the optimum set of boundary 
Table 3. Experimental results for Hand Gesture Identification for four different methods

\begin{tabular}{ccccc}
\hline Number of participants & G1 & G2 & G3 & G4 \\
\hline Method 1 & $57 \%$ & $57 \%$ & $57 \%$ & $57 \%$ \\
Method 2 & $65 \%$ & $65 \%$ & $65 \%$ & $65 \%$ \\
Method 3 & $60 \%$ & $60 \%$ & $60 \%$ & $60 \%$ \\
Method 4 & $99 \%$ & $99 \%$ & $99 \%$ & $99 \%$ \\
\hline
\end{tabular}

definitions given the desired class membership of each input case. This adaptive behavior makes the neural network a powerful paradigm for classification applications where the relationship between the inputs and the respective desired output classes is complex or difficult to resolve.

In this paper, pattern recognition of myoelectric signals is accomplished ANN through a state-driven method. In the first part of the experiment, RMS values of 4 recordings ( $s a, s b, s c$ and $s d$ ) for each subject were utilised to train the ANN classifier with back-propagation learning algorithm. The second part of the experiment (testing) was to verify the performance of the network. For that purpose a subset of all the input vectors different from the learning set (an independent data set) was selected. Performance was also monitored during the training phase in order to prevent overtraining of the network. The ANN consisted of two hidden layers with a total of 20 nodes. Back propagation gradient descent ANN training algorithm with sigmoid threshold was used for training and testing. During testing, the ANN with weight matrix generated during training was used to classify RMS of the muscle activity. The ability of the network to correctly classify the inputs against known subtle hand actions were used to determine the efficacy of the technique.

\section{RESULTS AND OBSERVATIONS}

The results of the experiment demonstrate the performance of the above described system. The results are tabulated in Tab. 3 and plotted in Fig. 5. To compare the performance of the system analysis on RAW sEMG and traditional ICA were performed. In traditional ICA method, mixing matrix was computed for each instance. The results demonstrate the ability of the semi-blind ICA in source separation and identification. The following four wrist actions and methods are labeled as below for displaying the results.

- Wrist flexion (G1)

- Wrist flexion towards little finger (G2)

- Wrist flexion towards thumb (G3) and

- Wrist and finger flexion together (G4).

- Method 1: Hand gesture Identification results on Raw EMG

- Method 2: Hand gesture Identification results using traditional ICA

- Method 3: Hand gesture Identification results using multi run ICA for worst mixing matrix (Lowest SIR)
- Method 4: Hand gesture Identification results using multi run ICA for best mixing matrix (Highest SIR)

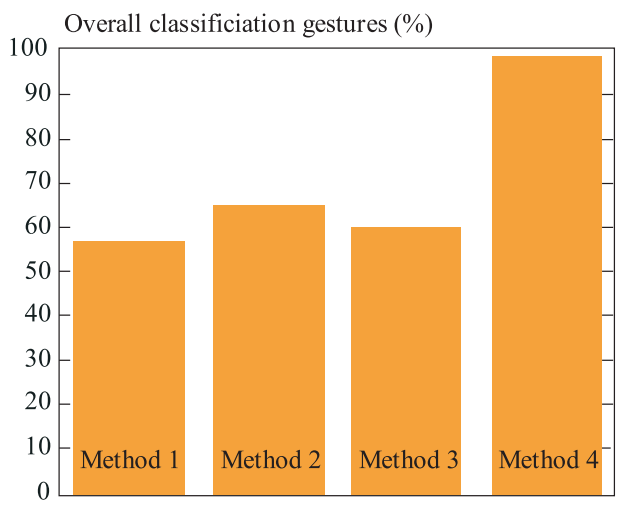

Fig. 5. The overall results showing hand gesture identification performance of MICA vs other methods

\section{DISCUSSIONS}

The proposed technique is capable of classifying small levels of muscle activity to identify four different wrist actions. Its base is using a combination of MICA, known anatomy and neural network configured for the individual. The technique has been tested with five volunteer participants and with experiments conducted on different days. The results indicate the ability of the system to perfectly recognise the hand gesture even though the muscle activity is very low and there are number of active muscles for each of the gestures.

The authors believe that the reason why this technique has succeeded where number of other similar techniques have failed is because the basis of this technique is to estimate the un-mixing matrix during training and maintaining this over the experiment ensuing the order and amplitude ambiguity is overcome. Further, other ICA based techniques are not suitable for near Gaussian signals and when signal-to-noise ratio is low and there is large crosstalk between different simultaneously active muscles. Use of BSS alone is not suitable for sEMG due to the nature of sEMG distribution and order ambiguity. Prior knowledge of the muscle anatomy combined with suitable BSS has overcome the above mentioned shortcomings.

\section{CONCLUSIONS AND FUTURE WORK}

This investigation has shown that a combination of the mixing matrix and network weights to classify the sEMG recordings in almost real-time. The results do indicate the ability of the system to work with the set of four different wrist movements selected. We are working on expanding the EMG gesture for increased levels of control. While, further work on the signal processing may make it possible to recognize multiple subtle gestures from a 
single muscle, it appears more practical to define a more extended interface using different controllers on various muscles (eg on both arms). Future work also shall include conducting experiments on inter-day and intra-day variations to verify the stability of the system and also developing a portable model for hand gesture recognition using semi blind ICA technique.

Overall, the purpose of this project is to develop new perceptual interfaces for human-computer-interaction based on hand gesture identification, and to investigate how such interfaces can complement or replace traditional interfaces based on keyboards, mice, remote controls, data gloves or speech. Applications fields for hand gestures analysis include control of consumer electronics, interaction with visualization systems, control of mechanical systems, and computer games.

One important benefit of such an HCI approach is that visual information makes it possible to communicate with computerized equipment at a distance, without a need for physical contact to the controlled target. Compared to speech commands, hand gestures are especially advantageous in noisy environments - particularly in situations where speech commands would be disturbed - as well as for communicating quantitative information and spatial relationships. Furthermore, the human user shall be enabled to control electronic systems in a quite natural manner, without requiring specialized external equipment.

\section{REFERENCES}

[1] ZARDoshti-KeRMAnI, M.-WHEELER, B. C.-BADIE, K.-HASHEMI, R. M.: EMG Feature Evaluation for Movement, Control of Upper Extremity Prostheses, IEEE Transaction on Rehabilitation Engineering 3 (1995), 324-233.

[2] HEFFTNER, G.-JAROS, G.: The Electromyogram (EMG) as a Control Signal for Functional Neuromuscular Stimulation, Part I: Autoregressive Modeling as a Mean of EMG Signature Discrimination, IEEE Transaction on Biomedical Engineering 35 No. 6 (1998), 228-235.

[3] BOnivento, C.-DAvalli, A.-FAnTuZzI, C.-SACCHETTI, R.-TERENZI, S. : Automatic Tuning of Myoelectric Prostheses, Journal of Rehab. Research and Develop 35 No. 3 (1998), 294-304.

[4] HUdGins, B.-PARKER, P.-SCOTT, R. N. : New Strategy for Multi-Function Myoelectric Control, IEEE Transaction on Biomedical Engineering 40 No. 1 yr1993, 82-94.

[5] CHANG, G. C.-KANG, W. J.-LUH, J. J.-CHENG, C. K.-LAI, J. S.-CHEN, J. J.-KUO, T. S.: Real-Time Implementation of Electromyogram Pattern Recognition as a Control Command of ManMachine Interface, Medical Engineering and Physics 18 No. 7 (1996), 529-537.

[6] ENGLEHART, K.-HUdGins, B. : A Robust, Real-Time Control Scheme for Multifunction Myoelectric Control, IEEE Trans. Biomed. Eng. 50 No. 7 (July 2003), 848-854.

[7] DOERSCHUK, P. C.-GUSTAFSON, D. E--WILLSKY, A. S. : Upper Extremity Limb Function Discrimination Using EMG Signal Analysis, IEEE Transactions on Biomedical Engineering 30 No. 1 (1983), 18-28.

[8] KERMANI, M. Z.-WHEELER, B. C.-BADIE, K.-HASHEMI, R. M. : EMG Feature Evaluation for Movement Control of Upper Extremity Prostheses, IEEE Transactions on Rehabilitation Engineering 2 (1995), 1267-1271.

[9] NAKAMURA, H.-YOSHIDA, M.-KOTANI, M.-AKAZAWA, K.-MORITANI, T.: The Application of Independent
Component Analysis to the Multichannel Surface Electromyographic Signals for Separation of Motor Unit Action Potential Trains, Journal of Electromyography and Kinesiology 14 No. 4 (Aug 2004), 423-432.

10] NAIK, G. R.-KUMAR, D. K.-SINGH, V. P.-PALANISWAMI, M. : SEMG for Identifying Hand Gestures using ICA, Workshop on Biosignal Processing and Classification at $2^{\text {nd }}$ International Conference on Informatics in Control, Automation and Robotics, Setubal, Portugal, Aug 2006, pp. 61-67.

11] CHLENZIG, J.-HUNTER, E.-JAIN, R. : Vision Based Hand Gesture Interpretation Using Recursive Estimation, In Twenty Eighth Asilomar Conference on Signals, Systems and Computers, volume 2, 1994, pp. 1267-1271.

12] REHG, J. M.-KANADE, D. T. : Vision-Based Hand Tracking for Human-Computer Interaction, in Proc. IEEE Workshop on Motion of Non-Rigid and Articulated Objects, 1994, pp. 16-22.

[13] SCHLENZIG, J.-HUNTER, E.-JAIN, R.: Vision Based Hand Gesture Interpretation Using Recursive Estimation, In Twenty-Eighth Asilomar Conference on Signals, Systems and Computers, 1994, pp. 1267-1271.

14] ChERON, G.-DRAYE, J. P.-BOURGEIOS, M.-LIBERT, G. : A Dynamic Neural Network Identification of Electromyography and Arm Trajectory Relationship during Complex Movements, IEEE Trans. Biomedical Engg. 43 (1996), 552-558.

15] PAVLOVIC, V. I.-SHARMA, R.-HUANG, T. S. : Visual Interpretation of Hand Gestures for Human Computer Interaction, IEEE Trans. Pattern Analysis and Machine Intelligence 19 (1997), 677-695.

16] BASMAJIAN—DELUCA, C.: Muscles Alive: Their Functions Revealed by Electromyography, $5^{\text {th }}$ Edn., Williams \& Wilkins, Baltimore, 1985.

[17] BELL, A.-SEJNOWSKI, T.: An information Maximisation Approach to Blind Separation and Blind Deconvolution, Neural Comput. 7 (1995), 1129-1159.

[18] HYVARINEN, A.-KARHUNEN, J.-OJA, E. : Independent Component Analysis, John Wiley, New York, 2001.

19] CICHOCKI, A.-AMARI, S. : Adaptive Blind Signal and Image Processing: Learning Algorithms and Applications, Wiley, New York, 2003.

20] HAIR, J. F.-BLACK, W. C.-BABIN, B. J.-ANDERSON, R. E.-TATHAM, R. L.: Multivariate Data Analysis, Prentice Hall, 2006.

Received 12 May 2009

Ganesh R. Naik received the BE degree in Electronics and communication engineering from the University of Mysore, India, in 1997, ME degree in communication and information engineering from Griffith University, Brisbane, Australia, in 2002, and $\mathrm{PhD}$ degree in the area of Digital signal processing from RMIT University, Melbourne, Australia, in 2009. His research interests include pattern recognition, Blind source separation techniques, Audio signal processing, biosignal processing and human computer interface. Presently he is serving as an academic and post doctoral fellow at the school of Electrical and computer engineering RMIT University, Australia.

Dinesh Kant Kumar received the BE degree in Electrical engineering from the Indian Institute of Technology (IIT) Madras, India, in 1982, and the PhD degree from IIT, Delhi, India, in 1990. He has worked in the engineering industry for over ten years in various capacities. Since 1996, he has been an Academic with RMIT University, Melbourne, Australia. His research interests include iterative signal processing, computer vision, and intelligent systems for application such as biometrics, human computer interface, and helping the disabled. 